\title{
ERRATA
}

\section{PARRONDO GAMES WITH SPATIAL DEPENDENCE}

\author{
[Fluctuation and Noise Letters, Vol. 11, No. 2 (2012) 1250004] \\ S. N. ETHIER \\ Department of Mathematics, University of Utah \\ 155 South 1400 East, Salt Lake City, Utah 84112, USA \\ ethier@math.utah.edu \\ JIYEON LEE \\ Department of Statistics, Yeungnam University \\ 214-1 Daedong, Kyeongsan, Kyeongbuk 712-749, South Korea \\ leejy@yu.ac.kr \\ Received 4 December 2011 \\ Revised 5 February 2012 \\ Published 5 October 2012 \\ Communicated by Zoltan Gingl
}

The following pages should appear as they are now. 
where rows and columns are labeled by $0,1,2,2^{\prime}, 3,4$, the two suggested methods being equivalent.

More generally, we can give a fairly explicit formula for $\overline{\boldsymbol{P}}$. First, define the function $s: \Sigma / \sim \mapsto\{0,1, \ldots, N\}$ by $s([\boldsymbol{x}]):=x_{1}+x_{2}+\cdots+x_{N}$; it counts the number of $1 \mathrm{~s}$ in each element of an equivalence class and is clearly well defined. Then

$$
\bar{P}([\boldsymbol{x}],[\boldsymbol{y}])= \begin{cases}N^{-1}\left(\sum_{i: x_{i}=0} q_{m_{i}(\boldsymbol{x})}+\sum_{i: x_{i}=1} p_{m_{i}(\boldsymbol{x})}\right) & \text { if }[\boldsymbol{y}]=[\boldsymbol{x}] \\ N^{-1} \sum_{i: x_{i}=1, \boldsymbol{x}^{i} \sim \boldsymbol{y}} q_{m_{i}(\boldsymbol{x})} & \text { if } s([\boldsymbol{y}])=s([\boldsymbol{x}])-1 \\ N^{-1} \sum_{i: x_{i}=0, \boldsymbol{x}^{i} \sim \boldsymbol{y}} p_{m_{i}(\boldsymbol{x})} & \text { if } s([\boldsymbol{y}])=s([\boldsymbol{x}])+1 \\ 0 & \text { otherwise }\end{cases}
$$

for all $[\boldsymbol{x}],[\boldsymbol{y}] \in \Sigma / \sim$. The first case in (8) follows because $\boldsymbol{x}^{i} \nsim \boldsymbol{x}$ for all $i$. Note also that, even if $s([\boldsymbol{y}])=s([\boldsymbol{x}]) \pm 1$, we may have $\bar{P}([\boldsymbol{x}],[\boldsymbol{y}])=0$; for example, $\bar{P}([001001],[010101])=0$. We say "fairly explicit" because the evaluation of $\overline{\boldsymbol{P}}$ still requires an enumeration of $\Sigma / \sim$, which can be time consuming.

\section{The Stationary Distribution}

The unique stationary distribution $\pi$ is too complicated to expect explicit formulas except for $3 \leq N \leq 6$. For $N=3$, we can find the unique stationary distribution for our eight-state chain by doing the same for a four-state chain. An invariant measure for the four-state chain is $\left(\rho_{0}, 3 \rho_{1}, 3 \rho_{2}, \rho_{3}\right)$, where

$$
\begin{aligned}
\rho_{0} & :=q_{0}\left(q_{1}+q_{2}\right) q_{3}, \\
\rho_{1} & :=p_{0}\left(q_{1}+q_{2}\right) q_{3}, \\
\rho_{2} & :=p_{0}\left(p_{1}+p_{2}\right) q_{3}, \\
\rho_{3} & :=p_{0}\left(p_{1}+p_{2}\right) p_{3},
\end{aligned}
$$

so an invariant measure for the eight-state chain is $\left(\rho_{0}, \rho_{1}, \rho_{1}, \rho_{2}, \rho_{1}, \rho_{2}, \rho_{2}, \rho_{3}\right)$. The stationary distribution follows by dividing each entry by the sum $\rho_{0}+3 \rho_{1}+3 \rho_{2}+\rho_{3}$. Incidentally, the $N=3$ case is the only case for which the stationary distribution is reversible ( $\overline{\boldsymbol{\pi}}$ in general, and $\boldsymbol{\pi}$ if $p_{1}=p_{2}$ ). A partial explanation is that $(6)$ is tridiagonal, that is, it corresponds to a birth-and-death chain.

For $N=4$ we can find the unique stationary distribution for our 16-state chain by doing the same for a six-state chain. An invariant measure for the six-state chain is $\left(\rho_{0}, 4 \rho_{1}, 4 \rho_{2}, 2 \rho_{2^{\prime}}, 4 \rho_{3}, \rho_{4}\right)$, where

$$
\begin{aligned}
& \rho_{0}:=q_{0}\left[2 q_{0} q_{3}+\left(q_{1}+q_{2}\right)^{2}\left(q_{0}+p_{3}\right)\right] q_{3}, \\
& \rho_{1}:=p_{0}\left[2 q_{0} q_{3}+\left(q_{1}+q_{2}\right)^{2}\left(q_{0}+p_{3}\right)\right] q_{3},
\end{aligned}
$$



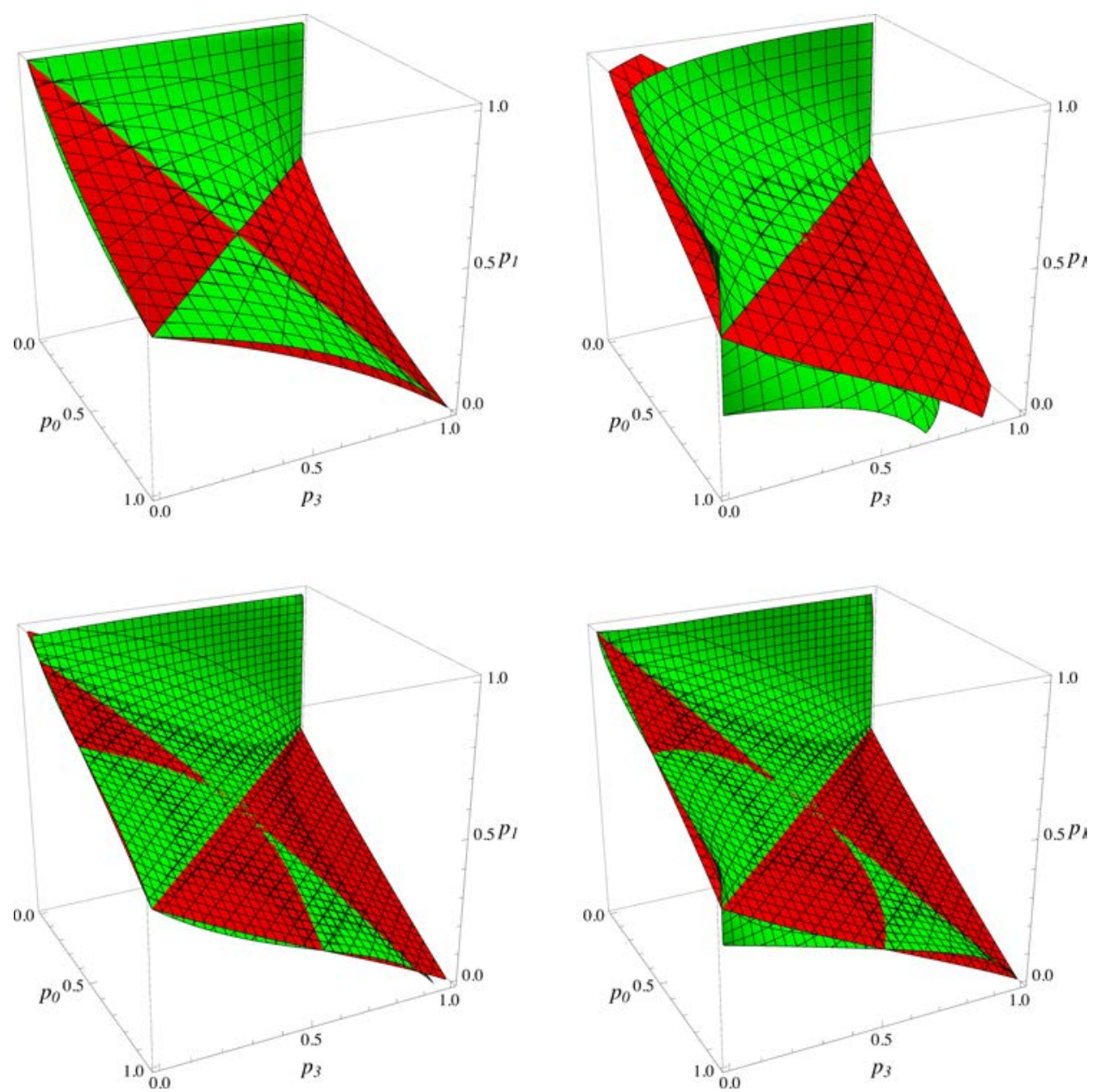

Fig. 1. When $N=3$ (upper left), $N=4$ (upper right), $N=5$ (lower left), or $N=6$ (lower right), the green (or light) surface is the surface $\mu_{B}=0$, and the red (or dark) surface is the surface $\mu_{C}=0$, both in the $\left(p_{0}, p_{3}, p_{1}\right)$ unit cube. The Parrondo region is the region on or below the green surface and above the red surface, while the anti-Parrondo region is the region on or above the green surface and below the red surface. (Assumptions: $p=1 / 2$ in game $A, p_{1}=p_{2}$ in games $B$ and $C$, and $\gamma=1 / 2$ in game $C$.)

For $i=1,2,3$, let $\boldsymbol{P}_{i}$ denote the $8 \times 8$ one-step transition matrix for the Markov chain in $\Sigma$ (with $N=3$ ) corresponding to player $i$ being chosen to play at each turn (as usual, the coin tossed depends on the status of the nearest neighbors). Then the one-step transition matrix $\boldsymbol{P}$ for Toral's game $B$ with $N=3$ can be defined as $\boldsymbol{P}:=(1 / 3)\left(\boldsymbol{P}_{1}+\boldsymbol{P}_{2}+\boldsymbol{P}_{3}\right)$. It can be shown that the stationary distribution of $\boldsymbol{P}$ coincides with the stationary distribution of $\boldsymbol{P}_{1} \boldsymbol{P}_{2} \boldsymbol{P}_{3}$ when $p_{1}=p_{2}$. The one-step transition matrix $\boldsymbol{P}_{1} \boldsymbol{P}_{2} \boldsymbol{P}_{3}$ corresponds to the game in which the three players 1, 2, 
Mihailović and Rajković [4] studied the presence of the Parrondo effect in the case of Toral's choice of the probability parameters, namely $p=1 / 2, p_{0}=1, p_{1}=$ $p_{2}=4 / 25$, and $p_{3}=7 / 10$ (actually, they took $p=0.499$ ). They obtained estimates of $\mu_{B}$ and $\mu_{C}$ using exact computations for $N=3$, unspecified analytic methods for

Table 2. Estimated volume of the Parrondo region. The integrated volume is obtained by integration or numerical integration and is rounded to six significant digits. For the Riemann sum approximation, the unit cube is divided into $(100)^{3}$ cubes of equal size, then the estimate is the proportion of cubes for which the Parrondo effect is present at the center point. The simulation estimate $\hat{p}$ is also based on $n=10^{6}$ points but here they are randomly chosen. The standard error is the square root of $\hat{p}(1-\hat{p}) / n$. (Assumptions: See the caption to Fig. 1.)

\begin{tabular}{rcccc}
\hline$N$ & $\begin{array}{c}\text { Integrated } \\
\text { volume }\end{array}$ & $\begin{array}{c}\text { Riemann sum } \\
\text { approximation to volume }\end{array}$ & $\begin{array}{c}\text { Simulation estimate } \\
\text { of volume }\end{array}$ & $\begin{array}{c}\text { Standard error } \\
\text { of simulation }\end{array}$ \\
\hline 3 & 0.0174361 & 0.017314 & 0.017336 & 0.0001305 \\
4 & 0.0293350 & 0.029199 & 0.029227 & 0.0001684 \\
5 & & 0.011275 & 0.011521 & 0.0001067 \\
6 & 0.010751 & 0.011090 & 0.0001047 \\
7 & 0.008327 & 0.008671 & 0.0000927 \\
8 & 0.007781 & 0.008028 & 0.0000892 \\
9 & 0.007060 & 0.007372 & 0.0000855 \\
10 & 0.006776 & 0.006952 & 0.0000831 \\
11 & & 0.006491 & 0.006791 & 0.0000821 \\
12 & 0.006356 & 0.006622 & 0.0000811 \\
13 & 0.006227 & 0.006492 & 0.0000803 \\
\hline
\end{tabular}

Table 3. Analysis of the Parrondo effect for Toral's choice of the probability parameters. Ellipses are intended to suggest that exact numbers have been truncated at six digits. The last two columns are rounded to six significant digits. (Assumptions: See the caption to Fig. 1.)

\begin{tabular}{|c|c|c|c|}
\hline$N$ & $\begin{array}{c}\text { Parrondo } p_{1} \text {-interval } \\
\text { when }\left(p_{0}, p_{3}\right)=(1,7 / 10)\end{array}$ & $\begin{array}{c}\mu_{B} \\
\text { at }\left(p_{0}, p_{1}, p_{3}\right)\end{array}$ & $\begin{array}{c}\mu_{C} \\
4 / 25,7 / 10)\end{array}$ \\
\hline 3 & $(0.195651 \cdots, 0.230769 \cdots]$ & -0.0909091 & -0.0183774 \\
\hline 4 & & 0.0799608 & 0.0171357 \\
\hline 5 & $(0.150762 \cdots, 0.162596 \cdots]$ & -0.00219465 & 0.00405176 \\
\hline 6 & $(0.149365 \cdots, 0.178102 \cdots]$ & -0.0189247 & 0.00463310 \\
\hline 7 & $(0.148884 \cdots, 0.155594 \cdots]$ & 0.00350598 & 0.00482261 \\
\hline 8 & $(0.148968 \cdots, 0.159157 \cdots]$ & 0.000698188 & 0.00479021 \\
\hline 9 & $(0.148967 \cdots, 0.162158 \cdots]$ & -0.00189233 & 0.00479036 \\
\hline 10 & $(0.148966 \cdots, 0.160394 \cdots]$ & -0.000332809 & 0.00479099 \\
\hline 11 & $(0.148966 \cdots, 0.160550 \cdots]$ & -0.000466527 & 0.00479089 \\
\hline 12 & $(0.148966 \cdots, 0.160793 \cdots]$ & -0.000676916 & 0.00479089 \\
\hline 13 & $(0.148966 \cdots, 0.160662 \cdots]$ & -0.000562901 & 0.00479089 \\
\hline 14 & $(0.148966 \cdots, 0.160669 \cdots]$ & -0.000569340 & 0.00479089 \\
\hline 15 & $(0.148966 \cdots, 0.160689 \cdots]$ & -0.000586184 & 0.00479089 \\
\hline 16 & $(0.148966 \cdots, 0.160680 \cdots]$ & -0.000578161 & 0.00479089 \\
\hline 17 & $(0.148966 \cdots, 0.160680 \cdots]$ & -0.000578345 & 0.00479089 \\
\hline 18 & $(0.148966 \cdots, 0.160681 \cdots]$ & -0.000579652 & 0.00479089 \\
\hline 19 & $(0.148966 \cdots, 0.160681 \cdots]$ & -0.000579095 & 0.00479089 \\
\hline
\end{tabular}


Table 4. Analysis of the Parrondo effect for a second point on the boundary of the unit cube and for a point in the interior. (Assumptions: See the caption to Fig. 1.)

\begin{tabular}{rccc}
\hline$N$ & $\begin{array}{c}\text { Parrondo } p_{1} \text {-interval } \\
\text { when }\left(p_{0}, p_{3}\right)=(7 / 10,0)\end{array}$ & $\begin{array}{c}\mu_{B} \\
\text { at }\left(p_{0}, p_{1}, p_{3}\right)=(7 / 10,17 / 25,0)\end{array}$ \\
\hline 3 & & 0.0710383 & 0.0297791 \\
4 & $(0.672790 \cdots, 0.807540 \cdots]$ & -0.0425713 & 0.00241457 \\
5 & $(0.657367 \cdots, 0.675341 \cdots]$ & 0.00257895 & 0.00818232 \\
6 & $(0.659797 \cdots, 0.699307 \cdots]$ & -0.0102930 & 0.00721881 \\
7 & $(0.659410 \cdots, 0.694010 \cdots]$ & -0.00722622 & 0.00736816 \\
8 & $(0.659472 \cdots, 0.695419 \cdots]$ & -0.00808338 & 0.00734464 \\
9 & $(0.659462 \cdots, 0.695052 \cdots]$ & -0.00784318 & 0.00734835 \\
10 & $(0.659463 \cdots, 0.695147 \cdots]$ & -0.00790952 & 0.00734776 \\
11 & $(0.659463 \cdots, 0.695122 \cdots]$ & -0.00789119 & 0.00734786 \\
12 & $(0.659463 \cdots, 0.695129 \cdots]$ & -0.00789624 & 0.00734784 \\
13 & $(0.659463 \cdots, 0.695127 \cdots]$ & -0.00789485 & 0.00734784 \\
14 & $(0.659463 \cdots, 0.695128 \cdots]$ & -0.00789523 & 0.00734784 \\
15 & $(0.659463 \cdots, 0.695127 \cdots]$ & -0.00789513 & 0.00734784 \\
16 & $(0.659463 \cdots, 0.695127 \cdots]$ & -0.00789516 & 0.00734784 \\
17 & $(0.659463 \cdots, 0.695127 \cdots]$ & -0.00789515 & 0.00734784 \\
18 & $(0.659463 \cdots, 0.695127 \cdots]$ & -0.00789515 & 0.00734784 \\
19 & $(0.659463 \cdots, 0.695127 \cdots]$ & -0.00789515 & 0.00734784 \\
\hline$N$ & Parrondo $p_{1}$-interval & $\mu_{B}$ & $\mu_{C}$ \\
& when $\left(p_{0}, p_{3}\right)=(1 / 10,3 / 4)$ & at $\left(p_{0}, p_{1}, p_{3}\right)=(1 / 10,3 / 5,3 / 4)$ \\
\hline 3 & $(0.611111 \cdots, 0.714285 \cdots]$ & -0.190476 & -0.00671141 \\
4 & $(0.584416 \cdots, 0.640975 \cdots]$ & -0.0858189 & 0.0108365 \\
5 & $(0.580262 \cdots, 0.616548 \cdots]$ & -0.0389980 & 0.0141217 \\
6 & $(0.579542 \cdots, 0.607387 \cdots]$ & -0.0183165 & 0.0147166 \\
7 & $(0.579415 \cdots, 0.603644 \cdots]$ & -0.00924232 & 0.0148223 \\
8 & $(0.579393 \cdots, 0.602063 \cdots]$ & -0.00528548 & 0.0148408 \\
9 & $(0.579390 \cdots, 0.601387 \cdots]$ & -0.00356984 & 0.0148441 \\
10 & $(0.579389 \cdots, 0.601097 \cdots]$ & -0.00282963 & 0.0148446 \\
11 & $(0.579389 \cdots, 0.600973 \cdots]$ & -0.00251155 & 0.0148447 \\
12 & $(0.579389 \cdots, 0.600920 \cdots]$ & -0.00237531 & 0.0148447 \\
13 & $(0.579389 \cdots, 0.600897 \cdots]$ & -0.00231709 & 0.0148448 \\
14 & $(0.579389 \cdots, 0.600887 \cdots]$ & -0.00229226 & 0.0148448 \\
15 & $(0.579389 \cdots, 0.600883 \cdots]$ & -0.00228169 & 0.0148448 \\
16 & $(0.579389 \cdots, 0.600881 \cdots]$ & -0.00227719 & 0.0148448 \\
17 & $(0.579389 \cdots, 0.600881 \cdots]$ & -0.00227528 & 0.0148448 \\
18 & $(0.579389 \cdots, 0.600880 \cdots]$ & -0.00227446 & 0.0148448 \\
19 & $(0.579389 \cdots, 0.600880 \cdots]$ & -0.00227412 & 0.0148448 \\
\hline & & &
\end{tabular}

